\title{
Costos cultivo de clones superiores de caoba (Swietenia macrophylla King) hasta 4 años de edad en Costa Rica
}

\author{
Cultivation costs of superior mahogany clones \\ (Swietenia macrophylla King) up to 4 years old in Costa Rica
}

Eugenio Corea-Arias ${ }^{1}$ (D) $\bullet$ Orlando Chinchilla-Mora ${ }^{1}$ (D) • Víctor Meza-Picado ${ }^{1}$ (D) $\bullet$ Carlos Ávila-Arias $^{1}$

Recibido: 15/10/2019 Aceptado: 19/05/2020Ｐublicado: 28/07/2020

\begin{abstract}
The cultivation of high-value timber species can represent a competitive alternative of land use, especially in agroforestry systems (AFS) where timber production costs are diluted throughout the entire production system. One of those species with high potential is mahogany; however, the information available on its cost structure is almost nil. The objective of the present investigation was to determine the strictly necessary cost items for the management of smallholder plantations (less than $5 \mathrm{ha}$ ), both for pure plantations and AFS. The cost of mahogany cultivation in pure plantations was very similar to other high-value species such as teak, and varied mainly due to site climate and weed control method used. The cost of growing mahogany in sites with a marked dry season was $6.8 \%$ higher than those that do not, due to the need to build and maintain firewalls and use water retainers gels. The cost of controlling the Hypsipyla grandella shoot borer represented $13.6 \%$ of the total cost of the production system; in addition, growing forest species in combination with agricultural crops was $125 \%$ less expensive than in pure plantations. The cost per tree in AFS up to four years decreased as the initial planting density increased, values between $\$ 2,095$ and \$ 1,139 were recorded for spacings between $4 \mathrm{~m} \times 4 \mathrm{~m}$ and $12 \mathrm{~m} \times 12 \mathrm{~m}$, respectively.
\end{abstract}

Key words: Silvicultural activities, shoot borer, AFS.

1. Instituto de Investigación y Servicios Forestales, Universidad Nacional (INISEFOR - UNA); Heredia, Costa Rica.

eugeniocorea@hotmail.com, orlando.chinchilla.mora@una.ac.cr, victor.meza.picado@una.ac.cr, carlos.avila.arias@una.ac.cr, 


\section{Resumen}

El cultivo de especies maderables de alto valor puede representar una alternativa competitiva del uso de la tierra, especialmente en sistemas agroforestales (SAF) donde algunos costos de producción de la madera se diluyen en la totalidad del sistema productivo. Una de esas especies con alto potencial es la caoba; sin embargo, es casi nula la información disponible sobre su estructura de costos. El objetivo de la presente investigación fue determinar los rubros de costos estrictamente necesarios para el manejo de plantaciones de pequeños productores (menos de $5 \mathrm{ha}$ ), tanto en plantaciones puras como SAF. El costo del cultivo de caoba en plantaciones puras fue muy similar al de otras especies de alto valor como la teca, varió principalmente en función del clima del sitio y método de control de arvenses utilizado. En sitios con estación seca marcada es 6,8 \% mayor que los que no la presentan, por la necesidad de construir y mantener cortafuegos y utilizar retenedores de agua al momento de plantar. En la presente investigación, el control del barrenador Hypsipyla grandella representó un $13,6 \%$ del costo total del sistema de producción, además cultivar especies forestales en combinación con cultivos agrícolas resultó $125 \%$ menos costoso que en plantaciones puras. El costo por árbol en SAF hasta los cuatro años disminuyó conforme aumentó la densidad inicial de siembra, se registraron valores entre $\$ 2095$ y \$1 139 para espaciamientos entre $4 \mathrm{~m} \mathrm{X} 4 \mathrm{~m}$ y $12 \mathrm{~m} \mathrm{X} 12$ $\mathrm{m}$, respectivamente.

Palabras clave: Actividades silviculturales, barrenador del tallo, SAF.

\section{Introducción}

En general, en Costa Rica se presenta una distribución de la tierra relativamente buena, al existir gran cantidad de fincas pequeñas en manos de productores agrícolas, forestales y agroforestales. En contraposición a lo anterior, el costo de la tierra y la mano de obra es alto en comparación con la gran mayoría de los países de la región. La anterior condición provoca que, el costo de producción de materia prima para la industria forestal, mediante plantaciones forestales puras, sea alto y, por ende, menos competitivo en comparación con otros países como Brasil, Chile, Colombia y Venezuela; principalmente por su producción a escala de especies de rápido crecimiento. Por otra parte, las plantaciones puras en Costa Rica también están en desventaja competitiva frente a otros usos alternativos de la tierra más rentables, al menos en el corto y mediano plazo, como por ejemplo la piña y cultivos de ciclo corto. La palma aceitera, el café, el cacao y los frutales también representan una fuerte competencia por la tierra.

Ante este panorama, el cultivo de especies maderables de alto valor en suelos de buena calidad, con material fenotípicamente superior y mediante una adecuada silvicultura clonal intensiva y sitio-específica, puede significar hacer competitiva ésta alternativa de producción. Lo anterior principalmente en sistemas agroforestales (SAF), donde los costos correspondientes al componente forestal son menores en comparación con plantaciones puras, al diluirse algunos de ellos en la totalidad del sistema productivo.

Una de esas especies con alto potencial es la caoba; sin embargo, es casi nula la información disponible sobre su estructura de costos, que abarque desde el establecimiento hasta su cosecha, tanto para plantación pura como en SAF. Dicha estimación de costos es indispensable para tomar decisiones sobre un plan de inversión y, desde luego, determinar la rentabilidad de su cultivo. Al respecto [1] apuntan que, las plantaciones de caoba presentaron indicadores financieros positivos, por lo que se puede considerar una buena opción de inversión, en el estado de Quintana Roo, la producción comercial con dicha especie.

Por la calidad y belleza de su madera, la caoba ha sido una de las especies forestales de mayor importancia y valor comercial en el trópico. Como consecuencia de esto, ha sufrido sobreexplotación en sus poblaciones naturales; lo que, sumado a la escasez de plantaciones comerciales en América Latina, genera una disminución importante en la oferta de su madera, con el consecuente aumento de su precio a nivel internacional. La principal limitante para el cultivo comercial de caoba en América es el ataque devastador del denominado "Barrenador de la familia Meliaceae" (Hypsipyla grandella Zeller).

Al respecto [2] apuntan que, una forma efectiva para prevenir y/o controlar ésta plaga es la aplicación de insecticidas en los brotes apicales tiernos de los árboles. No obstante, productores, técnicos y profesionales forestales no cuentan con información sobre los costos de dicho método químico. Es por ello que actores forestales asumen o intuyen que éste costo es muy alto, lo que provoca que las plantaciones de caoba no sean manejadas adecuadamente y por ende pierdan competitividad frente a otras de alto valor. Adicionalmente, existe una idea generalizada que todas las especies de maderas preciosas crecen lentamente, lo que también contribuye a que la caoba no sea tomada en cuenta para el establecimiento de sistemas de producción comercial.

Es en dicho escenario donde, la correcta estimación de los costos de un sistema de producción con caoba, 
establecido con material genético seleccionado y manejado adecuadamente, es fundamental para determinar el verdadero potencial económico (rentabilidad) de su cultivo. Lo anterior como parte un paquete tecnológico integral enfocado en reducir significativamente los turnos de corta y, por tanto, los costos en que se debe incurrir para alcanzar la altura comercial del fuste requerida.

Con el fin de minimizar barreras, ya sean tecnológicas, silviculturales $y / o$ financieras, para el cultivo comercial de la caoba, el Instituto de Investigación y Servicios Forestales de la Universidad Nacional de Costa Rica (INISEFOR - UNA) ha implementado, durante los últimos 13 años, proyectos para el mejoramiento genético y el desarrollo de métodos de cultivo de la especie, así como para la estimación de los costos de establecimiento y manejo hasta los 4 años de edad en diferentes condiciones ecológicas. Producto de las investigaciones se han identificado y multiplicado genotipos superiores, además se cuenta con un sistema silvicultural adaptado a las necesidades de la especie, donde se incluye desde luego el control de H. grandella [2], [3].

Con base en todo lo anterior, la presente investigación se planteó el objetivo de estimar aquellos rubros de costos estrictamente necesarios para el manejo de plantaciones puras de caoba de pequeños productores (menos de 5 ha), para dos sitios con condiciones climáticas distintas, y dichos costos compararlos con los de plantaciones puras de teca, adicionalmente estimarlos para sistemas agroforestales con caoba.

\section{Materiales y métodos}

Los rubros de costos que fueron aquí estimados se consideran los indispensables para el manejo de sistemas de producción con caoba de pequeños productores, quienes además de contar con una infraestructura básica (cercas, acceso, etc) también vigilan y administran personalmente la totalidad de su propiedad. Si se deseara extrapolar ésta información a sistemas de producción con mayor área, sería necesario agregar parámetros como el valor o alquiler de la tierra, establecimiento y mantenimiento de cercas, drenajes y caminos internos, cargas sociales para los trabajadores permanentes, así como gastos de vigilancia, gestión técnica y administración [1]; según corresponda en cada caso particular.

Como insumo base para la estimación realizada, se utilizó la información registrada en campo sobre materiales y mano de obra utilizados en todas las actividades de establecimiento y el manejo hasta los cuatro años. Lo anterior para dos sitios experimentales, el primero una plantación clonal de 1,5 hectáreas, densidad inicial de 1111 árboles por hectárea, localizada en un sitio muy lluvioso y con una estación seca muy corta o ausente (Horquetas), y el segundo también una plantación clonal de 0,9 hectáreas, densidad inicial de 1600 árboles por hectárea, en un sitio con una estación seca prolongada (Nicoya). Los precios de los materiales corresponden al mercado local y la mano de obra con un costo por jornal de $₫ 10$ 060, establecido por la legislación costarricense para el año 2018.

\section{Descripción de los sitios experimentales}

El sitio Horquetas se ubica en la finca del Campus de la Universidad Nacional en el cantón Sarapiquí, provincia Heredia. Se encuentra a una altitud de 80 m.s.n.m., con clima cálido y muy húmedo durante todo el año, sin una estación seca efectiva, evidenciado con precipitación media anual de $4050 \mathrm{~mm}$. La temperatura media anual es $26^{\circ} \mathrm{C}$ y topografía plana con pendientes menores al 2 $\%$. Al momento de establecer la plantación el sitio estaba en desuso, es decir cubierto densamente por arvenses, mayormente de especies de hoja ancha.

El segundo sitio se ubica en la finca del Colegio Técnico de Nicoya, cantón Nicoya, provincia Guanacaste. El sitio registra altitud de 130 m.s.n.m, precipitación media anual de $2126 \mathrm{~mm}$, con una estación lluviosa de mayo a noviembre y estación seca marcada, la temperatura media anual es de $26,2{ }^{\circ} \mathrm{C}$. La pendiente máxima del área experimental es $8 \%$. De la misma manera que el sitio anterior, previo a la plantación estaba en desuso, con arvenses mayormente de hoja ancha.

\section{Estimación costos cultivo de clones superiores de caoba en plantaciones puras}

Como se mencionó anteriormente, la información aquí usada para estimar costos en plantación pura se basó en una con espaciamiento inicial de $3 \mathrm{~m} \times 3 \mathrm{~m}$ y la otra de 2,5 $\mathrm{m} \times 2,5 \mathrm{~m}$ (sitio Horquetas y Nicoya, respectivamente). Dichos espaciamientos no se utilizan en plantaciones comerciales en Costa Rica hoy en día, máximo si se trata de material clonal. Por este motivo, los costos básicos que se presentan en este artículo se estimaron para una densidad inicial de 625 árb*ha-1 $^{-1}$ la que sí es muy utilizada a la fecha para material clonal. Adicionalmente a costos y actividades que son comúnmente asociados al establecimiento de la mayoría de especies forestales, para el cultivo intensivo de caoba se consideran los siguientes insumos y actividades:

Plantas: la producción clonal de caoba tarda hasta 5 meses en el invernadero del INISEFOR, lo que eleva el costo por cada árbol producido, con respecto a otras 
especies. El costo por árbol empleado para este estudio es de 550 colones.

Hoyado: los mismos fueron de $20 \mathrm{~cm}$ de diámetro y 25 $\mathrm{cm}$ de profundidad, es decir de mayor dimensión a lo normal para plantas producidas en pellets (Jiffy), esto con el fin de mejorar la estructura y aireación del suelo y promover así un buen crecimiento inicial de las raíces y el árbol en general.

Fertilización: $50 \mathrm{~g}$ de las fórmulas 10-30-10 ó 12-24-12 en el fondo del hoyo al momento de la siembra, igual en ambos sitios. En Horquetas adicionalmente $50 \mathrm{~g} \mathrm{y}$ $100 \mathrm{~g}$ por árbol a los 2 y 6 meses, respectivamente, con la fórmula 20-20-20 que además contiene magnesio y azufre. En Nicoya $50 \mathrm{~g}$ por árbol adicional a los 2 meses de la anterior fórmula; en este sitio a los 6 meses de plantado ya había iniciado el periodo seco por lo que no se fertilizó en ese momento. Al inicio de las lluvias en el segundo año (mayo) otra aplicación de $100 \mathrm{~g}$ por árbol y finalmente una de $200 \mathrm{~g}$ por árbol en agosto, ambos de la misma formulación 20-20-20.

Encalado: se consideró un costo de cal para corregir el $\mathrm{pH}$ y mejorar las condiciones de ambos sitios en cuanto a la fertilidad. En el presente estudio se consideró la aplicación $110 \mathrm{~kg}^{\star}$ ha-1 de cal en dos ocasiones durante el primer año, es decir un total de $220 \mathrm{~kg}^{\star} \mathrm{ha}^{-1}$.

Control del barrenador $H$. grandella: los árboles establecidos en campo se fumigaron cada dos semanas con insecticida cipermetrina, ésto hasta que alcanzaron $8 \mathrm{~m}$ de altura comercial. Es decir que, para la estimación de éste costo se consideró la aplicación durante 32 meses efectivos de crecimiento, es decir, meses con lluvia. Dependiendo de la calidad del sitio y de los clones plantados el tiempo podría ser menor, en algunos casos de apenas 24 meses efectivos, por lo que su costo podría ser menor. Solo se fumiga el brote terminal, para lo cual se utilizó una bomba de espalda de 18 litros, a la cual se le agregan extensiones para alcanzar alturas superiores a los 3,5 metros. La cipermetrina se aplicó a razón de $1 \mathrm{ml}$ por cada litro de agua. Para estimar el costo por hectárea de la bomba de espalda se consideró que se utiliza en 5 hectáreas durante 4 años. También se consideró el costo del adherente.

El tiempo por hectárea necesario para realizar las fumigaciones se estimó utilizando la siguiente fórmula:

$$
T E=B+(K \times F)+(T \times N)
$$

Donde:

$T E$ es el tiempo efectivo en minutos que se requiere para fumigar una hectárea.
$B$ es el tiempo en minutos que se utiliza para llenar una bomba de espalda de 18 litros. Se consideró constante y con una duración de 10 minutos.

$K$ es el tiempo promedio en minutos en que una persona camina $100 \mathrm{~m}$. El promedio general es de $5 \mathrm{~km}^{*} \mathrm{hora}^{-1}$. En las condiciones de plantaciones se estimó en 3,5 $\mathrm{km}^{*}$ hora $^{-1}$, los que significa 1,7 minutos para caminar una fila de $100 \mathrm{~m}$ sin fumigar los árboles.

$F$ es el número de filas de árboles por hectárea. Depende de la densidad y el arreglo espacial de distribución de los árboles. Para este estudio se consideró una distribución en cuadro ( $4 \times 4 \mathrm{~m}, 5 \times 5 \mathrm{~m}$, etc). Por, ejemplo, para un distanciamiento inicial de $4 \times 4 \mathrm{~m}$ el número de filas por hectárea es $25=$ 100/4.

$T$ es el tiempo en minutos que se tarda en aplicar el insecticida a un árbol sin tomar en cuenta el desplazamiento entre árbol y árbol. Varía según la altura del árbol. Se utilizaron tiempos promedio para cada año, con base en los datos recolectados en campo.

$N$ es el número de árboles por hectárea. Varía con el espaciamiento.

De esta forma entonces:

$B$ es el tiempo para llenar la bomba.

$K x F$ es el tiempo que se utiliza solamente para caminar todas las filas de árboles de una hectárea.

$T \times N$ es el tiempo que se utiliza solamente para fumigar todos los árboles de una hectárea

Control de arvenses: fundamental para el crecimiento inicial de la caoba. Para la estimación de sus costos se consideró la realización de chápeas en carriles de 1 $\mathrm{m}$ de ancho entre planta y planta, y se amplió a $1,5 \mathrm{~m}$ donde se ubica cada árbol, es decir $75 \mathrm{~cm}$ a cada lado. Con base en un distanciamiento inicial de $4 \mathrm{~m} \times 4 \mathrm{~m}$, el área controlada de arvenses es de $2700 \mathrm{~m}^{2 *} \mathrm{ha}^{-1}$, lo que representa solo un $27 \%$ del área total de una hectárea. El mantenimiento de los carriles facilita el paso y las labores durante las fumigaciones para el control de Hypsipyla grandella. Debido a las continuas precipitaciones en el sitio Horquetas, las chápeas debieron realizarse con mayor frecuencia que en Nicoya. En el caso de utilizar motoguadaña, durante el primer año se aplicó esta actividad cada 2 meses y en el segundo y tercero cada 2,5 meses. Al utilizar sólo herbicidas, las aplicaciones se hicieron cada 2,5 meses durante el primer año y cada 3 y 4 meses para el segundo y tercer año, respectivamente. Por su parte, en Nicoya las chápeas se realizaron únicamente durante la estación lluviosa; con motoguadaña cada 2 meses del año 1 al 3, mientras que 
mediante el uso de herbicidas 3 veces durante para los mismos tres años. Para el control químico de maleza se contempló la aplicación simultáneamente de pre y post emergentes. En ambos sitios se consideró una chapea durante el cuarto año, requerida para alguna actividad específica como medición o fertilización adicional entre otras. Para estimar el costo por hectárea del equipo (motoguadaña o bomba de espalda), se consideró utilizarlos en 5 hectáreas durante 4 años. También se incluyó en el cálculo el costo del combustible necesario, así como los herbicidas y adherentes.

Eliminación de bejucos: durante los primeros 6 ó 7 meses esta actividad se consideró parte de la limpieza de los carriles y/o rodajeas. Posterior a ese tiempo se incluyó en el cálculo su realización dos veces al año.

Podas: algunos árboles producen pocas ramas, generalmente a partir de los 5 ó $6 \mathrm{~m}$ de altura, pero estas son muy fáciles de desprender desde la base cuando aún están sin lignificar, mediante un gancho se tira de ellas suavemente. La primera poda se realizó a los 18 meses, de ahí en adelante cada 6 meses efectivos de lluvia, según cada sitio.

Cortafuegos: en el caso de Nicoya (aplica para sitios similares con estación seca prolongada) se consideró el establecimiento y mantenimiento de cortafuegos de $5 \mathrm{~m}$ de ancho durante todo el ciclo de la plantación; caso contrario Horquetas donde no es necesario. Este costo se calculó a partir de un corta fuegos de 5 metros de ancho para un terreno rectangular de 3 hectáreas (100 $\mathrm{m} \times 300 \mathrm{~m})$.

Gel retenedor de agua: muy útil y recomendado al momento de plantar en sitios donde el inicio de la estación lluviosa es irregular o incierto, como es el caso de Nicoya.

\section{Estimación y comparación de los costos básicos del cultivo de la caoba y la teca.}

Para realizar esta comparación se tomaron en cuenta aquellos rubros, cuyos costos, son esencialmente diferentes y particulares para cada una de las dos especies. Tales como el costo de los árboles, control de plagas, levantamiento de árboles (apuntalado), deshijas y podas. También se incluyen los costos comunes a ambas especies, ésto para estimar su peso relativo con respecto al total de costos estimado.

Para estimar los costos del apuntalado, deshija y podas de la teca se tomó como base la información presentada por [4], para varias plantaciones establecidas con una densidad inicial del 1111 árboles por hectárea, en diferentes sitios de Costa Rica. Los costos promedio de dicho estudio para la teca fueron ajustados para una densidad de 625 árb*ha $^{-1}$ y a los costos de los materiales y mano de obra del año 2018.

\section{Estimación de costo del cultivo de caoba en sistemas agroforestales}

Para tal fin se utilizó como base la información recolectada en el sitio Horquetas, por lo que los resultados generados aplican a sitios húmedos con una estación seca corta o ausente. En este estudio no se incluyen sistemas agroforestales denominados "silvopastoriles" ya que estos presentan una estructura de costos diferente.

Los rubros incluidos en éste apartado son capacitación inicial del productor, plantas, siembra, fertilización inicial, podas y control del barrenador hasta los 32 meses de edad. Estas son las erogaciones mínimas que el productor debe hacer cuando la caoba se combina con cultivos agrícolas. Los costos por preparación del terreno, el análisis de suelo, control de arvenses, control de hormigas cortadoras y la fertilización anual se le cargan al flujo de caja del cultivo asociado al componente forestal. Como los diferentes cultivos agrícolas requieren de distintas densidades de árboles para no afectar negativamente su desarrollo, se realizaron estimaciones para espaciamientos $4 \mathrm{~m} \times 4 \mathrm{~m}, 5 \mathrm{~m} \times 5,6 \mathrm{~m} \times 6 \mathrm{~m}$, $7 \mathrm{~m} \times 7 \mathrm{~m}, 8 \mathrm{~m} \times 8 \mathrm{~m}, 10 \mathrm{~m} \times 10 \mathrm{~m}$ y $12 \mathrm{~m} \times 12 \mathrm{~m}$. De esta forma se brindó una referencia más amplia para diferentes combinaciones mediante la extrapolación de la información generada. En todos los casos se estimaron los costos tanto por hectárea como por árbol.

\section{Resultados y discusión}

\section{Costo del cultivo de clones superiores de caoba en plantaciones puras}

En los cuadros 1, 2 y 3 se presentan los resultados, hasta los cuatro años de edad, para plantaciones puras con una densidad de 625 árb*ha $^{-1}$, tanto para Horquetas como para Nicoya. El costo total estimado para el cultivo de clones superiores de caoba hasta dicha edad oscila entre \$ 1546000 y \& 1752 000, ambos por hectárea (US\$ 2586-2930 ${ }^{*} \mathrm{ha}^{-1}$ ). Dicho rango se debe principalmente al clima del sitio y método empleado para el control de arvenses (Cuadro 1). Al respecto [5] agrega como aspectos que influyen en el costo definitivo la eficiencia y capacitación del personal, densidad inicial, intensidad de manejo, la topografía y forma del terreno, así como el acceso y la planificación.

Los resultados obtenidos son similares a los reportados para otras especies. En Panamá, [6] apuntan que para teca el costo total de establecimiento y manejo de los 
Cuadro 1. Costos anuales del cultivo de caoba en plantación pura hasta los 4 años de edad en Horquetas y Nicoya.

Table 1. Annual costs of mahogany cultivation in pure plantation up to 4 years old at Horquetas and Nicoya.

\begin{tabular}{|c|c|c|c|c|c|c|c|c|}
\hline \multirow[b]{2}{*}{ RUBRO } & \multicolumn{4}{|c|}{ HORQUETAS } & \multicolumn{4}{|c|}{ NICOYA } \\
\hline & $\begin{array}{l}\text { Chápeas con } \\
\text { motoguadaña }(\boldsymbol{\phi} \text { ha-1) }\end{array}$ & $\%$ & $\begin{array}{l}\text { Chápeas con } \\
\text { herbicida }\left(\boldsymbol{\phi} \mathrm{ha}^{-1}\right)\end{array}$ & $\%$ & $\begin{array}{c}\text { Chápeas con } \\
\text { motoguadaña ( } \$ \\
\text { ha-1) }\end{array}$ & $\%$ & $\begin{array}{c}\text { Chápeas con } \\
\text { herbicida }\left(\boldsymbol{\phi} \text { ha }^{-1}\right)\end{array}$ & $\%$ \\
\hline Establecimiento & 606360 & 36,8 & 606360 & 39,2 & $\left(\Phi^{\star} h a-1\right)$ & $\%$ & 641390 & 38,7 \\
\hline Manejo año 1 & 400682 & 24,3 & 370432 & 24,0 & 316543 & 18,2 & 307298 & 18,5 \\
\hline Manejo año 2 & 299126 & 18,1 & 263891 & 17,1 & 325755 & 18,8 & 291003 & 17,5 \\
\hline Manejo año 3 & 270810 & 16,4 & 241890 & 15,6 & 211460 & 12,2 & 183247 & 11,0 \\
\hline Manejo año 4 & 72570 & 4,4 & 63740 & 4,1 & 239975 & 13,8 & 236379 & 14,3 \\
\hline \multirow{2}{*}{ Total } & 1649548 & \multirow{2}{*}{100} & 1546313 & \multirow{2}{*}{100} & 1752627 & \multirow{2}{*}{100} & 1659317 & \multirow{2}{*}{100} \\
\hline & US\$ 2758 & & US\$ 2586 & & US\$ 2930 & & US\$ 2775 & \\
\hline
\end{tabular}

Estimaciones realizadas para 625 árb*ha-1 $^{-1}$

primeros cuatro años de US\$ 3222 ha $^{-1}$, es decir un $9,9 \%$ superior a los aquí generados. Por su parte, [1] reportaron costos de establecimiento y manejo de los primeros años significativamente superiores a los del presente estudio, pero para una densidad inicial de siembra de 1111 árb*ha $^{-1}$.

En promedio, los costos totales estimados para Nicoya

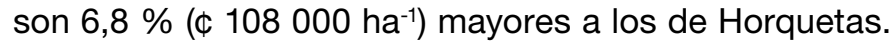
Esto se debe principalmente al establecimiento y mantenimiento de líneas corta fuegos, así como al uso de retenedores de agua, ambos rubros indispensables en sitios con estación seca marcada y peligro latente de incendios.

En el sitio Nicoya los costos anuales están distribuidos más uniformemente entre los primeros cuatro años que en Horquetas. Lo anterior, principalmente, a que en Nicoya se necesita esa cantidad de tiempo para alcanzar 32 meses efectivos de crecimiento, debido a la ocurrencia de la estación prolongada. Por este motivo, el control del barrenador se debe realizar durante aproximadamente 7,5 meses cada año durante esos cuatro años. En el caso del sitio Horquetas los 32 meses efectivos de crecimiento se logran en 2,7 años, momento a partir del cual se suspenden las fumigaciones con cipermetrina, con lo que se concentra ese rubro en los primeros años. De igual forma y por el mismo motivo, el número de chapeas anuales en Nicoya también se distribuye más uniformemente entre años, mientras que en Horquetas se concentra en el mismo período de tiempo que las fumigaciones.

El costo de establecimiento varía entre \ 606000 ha $^{-1}$ y $\$ 641.000$ ha $^{-1}$, representa entre el 36,8 \% y 39,2
$\%$ del total (Cuadro 1). Se convierte en el año donde se registran mayores cifras para cualquiera de los escenarios propuestos, seguido por los tres primeros años de manejo. [7] reportan un porcentaje mayor (46-53 \%) correspondiente al año de establecimiento; no obstante, incluyen gastos de administración, entre otros, lo que eleva dicho costo. Por su parte, autores como [8] confirman que, el establecimiento de las plantaciones forestales el rubro en el que se desembolsa la mayor cantidad de dinero por año; por otro lado, el mantenimiento durante los primeros dos o tres años le siguen en costos, posteriormente se estabilizan con picos en los años donde se realizan actividades como raleos y podas, además de los años de cosechas donde también se elevan.

El costo promedio estimado para el control de arvenses en ambos sitios mediante la motoguadaña es $₫ 287000$ ${ }^{*} \mathrm{ha}^{-1}$, mientras que si se utilizan herbicidas se reduce a $\phi$ $18800{ }^{*} \mathrm{ha}^{-1}$ (Cuadro 2), es decir una diferencia promedio de $52,6 \%$ entre ambos métodos. Sin embargo, a nivel general, si las chápeas se realizan con motoguadaña el costo promedio total por hectárea (\$1701 000) es 6,1\% mayor que el costo total si las chápeas se efectúan con herbicidas ( $\$ 1603$ 000), principalmente debido a que las chápeas con motoguadaña se realizan con mayor frecuencia que si se aplicaran herbicidas. El costo de las mismas representan del $11 \%$ al $18 \%$ del costo total por hectárea, dependiendo del sitio y del método de control utilizado, para un promedio de $14,3 \%$. De la misma manera, [9] encontró que los costos de las chapeas fueron menores usando con motoguadaña, con un promedio de tres jornales por hectárea, eso sí para chapea general y no únicamente en los carriles como aquí se plantea. 
Cuadro 2. Costos totales del cultivo de caoba en plantación hasta los 4 años para cada rubro.

Table 2. Total costs of mahogany cultivation in plantation up to 4 years old for each item.

\begin{tabular}{|c|c|c|c|c|c|c|c|c|}
\hline \multirow[b]{2}{*}{ RUBRO } & \multicolumn{4}{|c|}{ HORQUETAS } & \multicolumn{4}{|c|}{ NICOYA } \\
\hline & $\begin{array}{l}\text { Chápeas con } \\
\text { motoguadaña } \\
\qquad\left(\mathbf{\phi} \mathrm{ha}^{-1}\right)\end{array}$ & $\%$ & $\begin{array}{l}\text { Chápeas } \\
\text { con } \\
\text { herbicida } \\
\left(\$ \text { ha }^{-1}\right)\end{array}$ & $\%$ & $\begin{array}{l}\text { Chápeas con } \\
\text { motoguadaña } \\
\qquad\left(\$ \text { ha }{ }^{-1}\right)\end{array}$ & $\%$ & $\begin{array}{c}\text { Chápeas con } \\
\text { herbicida } \\
\left(\mathbf{\phi} \text { ha }^{-1}\right)\end{array}$ & $\%$ \\
\hline Asistencia técnica & 100.000 & 6,0 & 100000 & 6,5 & 100000 & 5,7 & 100000 & 6,0 \\
\hline Análisis de suelo & 15000 & 0,9 & 15000 & 1,0 & 15000 & 0,9 & 15000 & 0,9 \\
\hline \multicolumn{9}{|l|}{ Preparación sitio } \\
\hline y siembra & 196220 & 11,9 & 196220 & 12,7 & 231250 & 13,2 & 231250 & 13,9 \\
\hline Plantas & 357500 & 21,7 & 357500 & 23,1 & 357500 & 20,4 & 357500 & 21,5 \\
\hline $\begin{array}{l}\text { Chápeas años } 1 \\
\text { al } 4\end{array}$ & 296605 & 18,0 & 193370 & 12,5 & 276530 & 15,8 & 183220 & 11,0 \\
\hline Rodajeas manuales & 80480 & 4,9 & 80480 & 5,2 & 80480 & 4,6 & 80480 & 4,9 \\
\hline Control barrenador & 224188 & 13,6 & 224188 & 14,5 & 226802 & 12,9 & 226802 & 13,7 \\
\hline Control de bejucos & 40240 & 2,4 & 40240 & 2,6 & 40240 & 2,3 & 40240 & 2,4 \\
\hline Podas & 20120 & 1,2 & 20120 & 1,3 & 15090 & 0,9 & 15090 & 0,9 \\
\hline Control de hormigas & 64000 & 3,9 & 64000 & 3,9 & 64000 & 3,6 & 64000 & 3,9 \\
\hline $\begin{array}{c}\text { Fertilizaciones, } \\
\text { encalado }\end{array}$ & 255195 & 15,5 & 255195 & 16,5 & 255195 & 14,5 & 255195 & 15,4 \\
\hline Corta fuegos & - & 0 & - & 0 & 90540 & 5,2 & 90540 & 5,5 \\
\hline Total & 1649548 & 100 & 1546313 & 100 & 1752627 & 100 & 1659317 & 100 \\
\hline
\end{tabular}

Estimaciones realizadas para 625 árb*ha-1

Cuadro 3. Resumen del costo de materiales y mano de obra del cultivo de caoba en plantación hasta los 4 años en Horquetas y Nicoya.

Table 3. Summary of materials and workforce's cost of mahogany cultivation in plantation up to 4 years old at Horquetas and Nicoya.

\begin{tabular}{|c|c|c|c|c|c|c|c|c|}
\hline \multirow[b]{2}{*}{ RUBRO } & \multicolumn{4}{|c|}{ HORQUETAS } & \multicolumn{4}{|c|}{ NICOYA } \\
\hline & $\begin{array}{l}\text { Chápeas con } \\
\text { motoguadaña }\left(\$ \text { ha }^{-1}\right)\end{array}$ & $\%$ & $\begin{array}{c}\text { Chápeas con } \\
\text { herbicida }\left(\$ \text { ha } \mathbf{h}^{-1}\right)\end{array}$ & $\%$ & $\begin{array}{c}\text { Chápeas con } \\
\text { motoguadaña (\$ } \\
\left.\text { ha }^{-1}\right)\end{array}$ & $\%$ & $\begin{array}{l}\text { Chápeas con } \\
\text { herbicida }\left(\mathbf{\phi} \text { ha }{ }^{-1}\right)\end{array}$ & $\%$ \\
\hline $\begin{array}{l}\text { Asistencia } \\
\text { técnica }\end{array}$ & 100000 & 6,1 & 100000 & 6,5 & 100000 & 5,7 & 100000 & 6,0 \\
\hline $\begin{array}{l}\text { Análisis de } \\
\text { suelo }\end{array}$ & 15000 & 0,9 & 15000 & 1,0 & 15000 & 0,9 & 15000 & 0,9 \\
\hline $\begin{array}{l}\text { Árboles y } \\
\text { materiales }\end{array}$ & 711640 & 43,1 & 581340 & 44,0 & 754520 & 43,6 & 723540 & 43,7 \\
\hline Mano de obra & 822908 & 49,9 & 749973 & 48,5 & 873107 & 49,8 & 817777 & 49,4 \\
\hline Manejo año 4 & 72570 & 4,4 & 63740 & 4,1 & 239975 & 13,8 & 236379 & 14,3 \\
\hline Total & 1649548 & 100 & 1546313 & 100 & 1752627 & 100 & 1659317 & 100 \\
\hline
\end{tabular}

Estimaciones realizadas para 625 árb*ha-1 $^{-1}$

Desde el punto de vista ambiental y social, resulta de gran importancia que sea solamente un 6,1 \% mayor el costo de emplear la motoguadaña en el control de arvenses. Lo anterior se convierte en básico para la toma de decisiones hacia minimizar, o evitar del todo, los problemas asociados al uso de herbicidas y justificar la mano de obra adicional, y el consecuente aumento en los ingresos de los trabajadores que operan dicho equipo. No obstante, dependerá de la disponibilidad de todos los recursos necesarios para llevar a cabo ésta actividad 
Cuadro 4. Costos control del barrenador Hypsipyla grandella en plantación pura de caoba hasta los 4 años en Horquetas y Nicoya.

Table 4. Control costs of Hypsipyla grandella shoot borer in mahogany pure plantation up to 4 years old at Horquetas and Nicoya.

\begin{tabular}{|ccccc|}
\hline \multirow{2}{*}{ RUBRO } & \multicolumn{2}{c}{ HORQUETAS } & \multicolumn{2}{c|}{ NICOYA } \\
\cline { 2 - 5 } & $\begin{array}{c}\text { Costo } \\
\left(\Phi^{*} \text { ha }^{-1}\right)\end{array}$ & $\%$ & $\begin{array}{c}\text { Costo } \\
\left(\Phi^{*} \text { ha }^{-1}\right)\end{array}$ & $\%$ \\
\hline Mano de obra & 199188 & 88,8 & 201202 & 88,7 \\
\hline Insecticida y adherente & 15000 & 6,7 & 15600 & 6,9 \\
\hline Bomba de fumigación & 10000 & 4,5 & 10000 & 4,4 \\
\hline Total & 224188 & 100 & 226802 & 100 \\
\hline Estimaciones realizadas para 625 árb*ha ${ }^{-1}$ & & \\
\hline
\end{tabular}

Cuadro 5. Diferencias en los costos de cultivo entre la caoba y la teca en plantación pura.

Table 5. Differences in crop costs between mahogany and teak in pure plantation.

\begin{tabular}{|c|c|c|c|c|}
\hline \multirow[b]{2}{*}{ RUBRO } & \multicolumn{2}{|c|}{ CAOBA } & \multicolumn{2}{|l|}{ TECA } \\
\hline & $\begin{array}{l}\text { Chápeas } \\
\text { con } \\
\text { herbicida } \\
\left(\Phi^{*} \text { ha }^{-1}\right)\end{array}$ & $\%$ & $\begin{array}{c}\text { Chápeas con } \\
\text { herbicida } \\
\left(\Phi^{\star} h a^{-1}\right)\end{array}$ & $\%$ \\
\hline Plantas & 357500 & 21,5 & 211250 & 12,7 \\
\hline $\begin{array}{c}\text { Control de } H . \\
\text { grandella } 32 \\
\text { meses }\end{array}$ & 226802 & 13,7 & - & - \\
\hline $\begin{array}{c}\text { Apuntalado, } \\
\text { deshijas y } \\
\text { podas hasta el } \\
\text { año } 12\end{array}$ & 15090 & 0,9 & 392340 & 23,6 \\
\hline $\begin{array}{l}\text { Otros costos } \\
\text { comunes hasta } \\
\text { el año } 4\end{array}$ & 1059925 & 63,9 & 1059925 & 63,7 \\
\hline Total & 1659318 & 100 & 1663515 & 100 \\
\hline
\end{tabular}

silvicultural. Ligado a esto, el costo promedio del control de arvenses en Horquetas es ligeramente mayor que en Nicoya (6,5\%), principalmente por no presentar una estación seca marcada como en el segundo sitio.

De todos los rubros considerados en el Cuadro 2, el costo de las plantas es el mayor ( $\$ 357500{ }^{*} \mathrm{ha}^{-1}$, USD\$ $0,90 *^{*}$ rb $^{-1}$ ) al representar en promedio un $57 \%$ del establecimiento. No obstante, [1] reportaron un costo de $\$ 1,7$ por planta, lo que es un monto mayor al utilizado en el cálculo del presente estudio. Los árboles fueron producidos en un invernadero ubicado a $1250 \mathrm{msnm}$, las condiciones ambientales que ahí se encuentran repercuten en periodos prolongados para tener las plantas lista para campo, lo que finalmente provoca el incremento en su costo de producción.

Además se identifican otros rubros de costo como importantes en el sistema de producción, entre ellos fertilización, chápeas, control de $H$. grandella y preparación del sitio, los cuales representan en promedio $15,5 \%, 14,3 \%, 13,7 \%$ y $12,9 \%$ del costo total aquí reportado, respectivamente. Dichas actividades son fundamentales para alcanzar la calidad y la altura deseada del fuste comercial en el menor tiempo posible.

Cuando las actividades son realizadas por mano de obra ocasional, el costo de este rubro representa entre 48,5 $\%$ y $49,9 \%$ del costo total de una hectárea (Cuadro 3 ). En el caso de plantaciones grandes, donde se tiene una relación laboral permanente con los trabajadores es necesario agregar el costo de las cargas sociales.

\section{Costo control del barrenador Hypsipyla grandella y su importancia relativa en plantaciones puras}

De acuerdo a los resultados obtenidos, el costo de ésta actividad no es tan alto como, a priori, se esperaba (Cuadro 4). Hasta los 4 años de edad, en Horquetas se registró un monto total de $₫ 224188^{*} \mathrm{ha}^{-1}$, mientras que en Nicoya \& $226802{ }^{*} \mathrm{ha}^{-1}$, es decir muy similares en ambos sitios. Representa entre el $12,9 \%$ y $14,5 \%$ del costo total para los casos aquí analizados. El principal componente de este costo es la mano de obra, con un $88,7 \%$ del total de la actividad.

Con base en los resultados generados, el costo de ésta actividad no debería ser un factor limitante para que el cultivo de la caoba sea rentable. Es claro que los beneficios obtenidos en términos de calidad y productividad de la plantación, si se realizan correctamente, justifican ver éste rubro como una inversión y no tanto un costo en sí mismo. No obstante, es necesario realizar análisis financieros de distintos escenarios de sistemas de producción, con el objetivo de estimar el costo/beneficio de ésta actividad silvicultural para cada uno. Según la experiencia generada y los resultados aquí obtenidos, el principal asunto por resolver, en lo que se refiere al control del barrenador mediante la aplicación del insecticida, no parece ser su costo sino la planificación y disciplina necesarias para realizar las fumigaciones correctamente y con la frecuencia requerida. En este sentido, se requiere fomentar mayor cultura forestal en los productores para que visualicen su sistema de producción como el cultivo comercial de madera. 
Cuadro 6. Costos básicos anuales estimados para producción de caoba en combinación con cultivos.

Table 6. Estimated annual basic costs of mahogany production in combination with crops.

\begin{tabular}{|ccc|}
\hline \multirow{2}{*}{ Rubro } & \multicolumn{2}{c|}{ Costo } \\
\cline { 2 - 3 } & $\left(\Phi^{*}\right.$ ha $\left.^{-1}\right)$ & $\%$ \\
\hline Establecimiento & 311215 & 61,9 \\
\hline Manejo año 1 & 47908 & 9,5 \\
\hline Manejo año 2 & 69588 & 13,8 \\
\hline Manejo año 3 & 70294 & 14,0 \\
\hline Manejo año 4 & 4025 & 0,8 \\
\hline Total & 503034 & 100 \\
\hline
\end{tabular}

Estimaciones realizadas para 400 árb*ha-1

Cuadro 7. Costos básicos estimados por rubro para el cultivo de caoba en combinación con cultivos.

Table 7. Estimated basic costs per item for mahogany cultivation in combination with crops.

\begin{tabular}{|ccc|}
\hline \multirow{2}{*}{ Rubro } & \multicolumn{2}{c|}{ Costo } \\
\cline { 2 - 3 } Capacitación inicial & $\left(\phi^{*}\right.$ ha- 1$)$ & $\%$ \\
\hline Siembra & 25000 & 5,0 \\
\hline Plantas & 52815 & 10,5 \\
\hline Control del & 225500 & 44,8 \\
\hline barrenador & 175723 & 34,9 \\
\hline Podas & 16096 & 3,2 \\
\hline Fertilización inicial & 7900 & 1,6 \\
\hline Total & 503034 & 100 \\
\hline
\end{tabular}

Estimaciones realizadas para 400 árb*ha*

Cuadro 8. Costo mano de obra y su importancia relativa para el cultivo de caoba en combinación con cultivos.

Table 8. Labor cost and its relative importance for the cultivation of mahogany in combination with crops.

\begin{tabular}{|ccc|}
\hline \multirow{2}{*}{ Rubro } & \multicolumn{2}{c|}{ Costo } \\
\cline { 2 - 3 } & $\left(\Phi^{*}\right.$ ha $\left.{ }^{-1}\right)$ & $\%$ \\
\hline Capacitación inicial & 25000 & 5,2 \\
\hline $\begin{array}{c}\text { Plantas }+ \\
\text { materiales }\end{array}$ & 254400 & 50,4 \\
\hline Mano de obra & 223634 & 42,4 \\
\hline Total & 503034 & 100 \\
\hline
\end{tabular}

Estimaciones realizadas para 400 árb*ha*
Comparación entre el costo de cultivo de caoba y teca

La teca (Tectona grandis) es una de principales alternativas para cultivar en lugar de la caoba. Se trata de la especie más plantada en Costa Rica [10] y en general en todo el trópico, debido a su relativamente rápido crecimiento y a la calidad y valor de su madera, lo que la ha llevado a tener precio internacional de mercado. Además, ambas especies comparten en gran medida las áreas geográficas y sitios donde pueden ser plantadas. Por lo anterior, es justificable utilizar teca como referencia para comparar los costos de producción de la caoba.

En el Cuadro 5 se presentan los rubros con las principales diferencias entre ambas especies en cuanto a los costos de producción. Dichas estimaciones fueron realizadas para el sitio Nicoya y se tomó como base, para ambas especies, el control de arvenses con herbicidas.

Los costos básicos de establecimiento y manejo de la teca y la caoba son prácticamente iguales; no obstante, los de la caoba serán menores en la medida que se logre minimizar el valor de las plantas. Las diferencias de costos en rubros particulares de cada especie se compensan en el balance general. Al respecto, teca tiene un alto costo para las labores de apuntalamiento, es decir el levantamiento de árboles jóvenes caídos producto de episodios de viento fuerte, así como deshijas y podas; mientras que en la caoba, se le debe sumar al mayor costo de las plantas, el control del barrenador. Los costos comunes de producción son prácticamente los mismos para ambas especies.

Los resultados obtenidos apuntan que, la caoba representa una alternativa real y accesible para producir madera de alto valor. Entonces, la decisión final de cuál de las dos plantar dependerá del objetivo de producción, mercado (consumidores finales) y el análisis completo de rentabilidad de cada una de ellas para cada caso en particular, donde se debe tomar muy en cuenta diferencias en el turno de corta y valor de las cosechas intermedias y la final. Actualmente, el precio internacional de la madera de caoba es superior al de la teca; no obstante, es necesario valorar si la superioridad en crecimiento de clones de teca pueden compensar o nó dichas diferencias de precio.

\section{Costos de producción de la caoba en combinación con cultivos agrícolas - SAF}

Para ésta estimación se utilizó como base la información generada en el sitio Horquetas. Los rubros contenidos en el Cuadro 7 son considerados las erogaciones mínimas que un productor debe hacer para cultivar árboles de caoba en sistemas agroforestales - SAF. Se asume también que los costos correspondientes a la preparación 
Cuadro 9. Costos básicos del cultivo de caoba en combinación con cultivos para diferentes espaciamientos inicales, por hectárea y árbol.

Table 9. Basic costs of mahogany cultivation in combination with crops for different initial spacing, per hectare and tree.

\begin{tabular}{|c|c|c|c|c|c|c|c|c|}
\hline $\begin{array}{l}\text { Densidadn } \\
\text { inicial } \\
\left(\text { arb*ha }^{-1}\right)\end{array}$ & $\begin{array}{c}\text { Espaciamiento } \\
\text { entre árboles } \\
(\mathrm{m} \times \mathrm{m})\end{array}$ & $\begin{array}{l}\text { Capacitación } \\
\text { inicial (CAP) }\end{array}$ & $\begin{array}{l}\text { Plantas y } \\
\text { materiales } \\
\text { (MAT) }\end{array}$ & $\begin{array}{l}\text { Mano de } \\
\text { obra (MO) }\end{array}$ & $\begin{array}{l}\text { Costo } \\
\left(\Phi^{\star} h a^{-1}\right)\end{array}$ & $\begin{array}{c}\text { Costo } \\
\text { MAT + CAP } \\
\left(\Phi^{*} a ́ r b-1\right)\end{array}$ & $\begin{array}{l}\text { Costo } \\
\text { MO } \\
\left(\Phi^{\star} \text { árb }^{-1}\right)\end{array}$ & $\begin{array}{c}\text { Costo } \\
\left(\Phi^{\star} \text { árb }{ }^{-1}\right)\end{array}$ \\
\hline 625 & $4 \times 4$ & 25.000 & 395140 & 291539 & 711679 & 672 & 467 & 1139 \\
\hline 400 & $5 \times 5$ & 25.000 & 254400 & 223634 & 503034 & 699 & 559 & 1258 \\
\hline 278 & $6 \times 6$ & 25.000 & 180030 & 161865 & 366895 & 738 & 582 & 1320 \\
\hline 204 & $7 \times 7$ & 25.000 & 134629 & 130981 & 290610 & 783 & 642 & 1425 \\
\hline 156 & $8 \times 8$ & 25.000 & 105481 & 108547 & 239028 & 836 & 696 & 1532 \\
\hline 100 & $10 \times 10$ & 25.000 & 72575 & 85208 & 182783 & 976 & 852 & 1828 \\
\hline 70 & $12 \times 12$ & 25.000 & 53459 & 68207 & 146665 & 1121 & 974 & 2095 \\
\hline
\end{tabular}
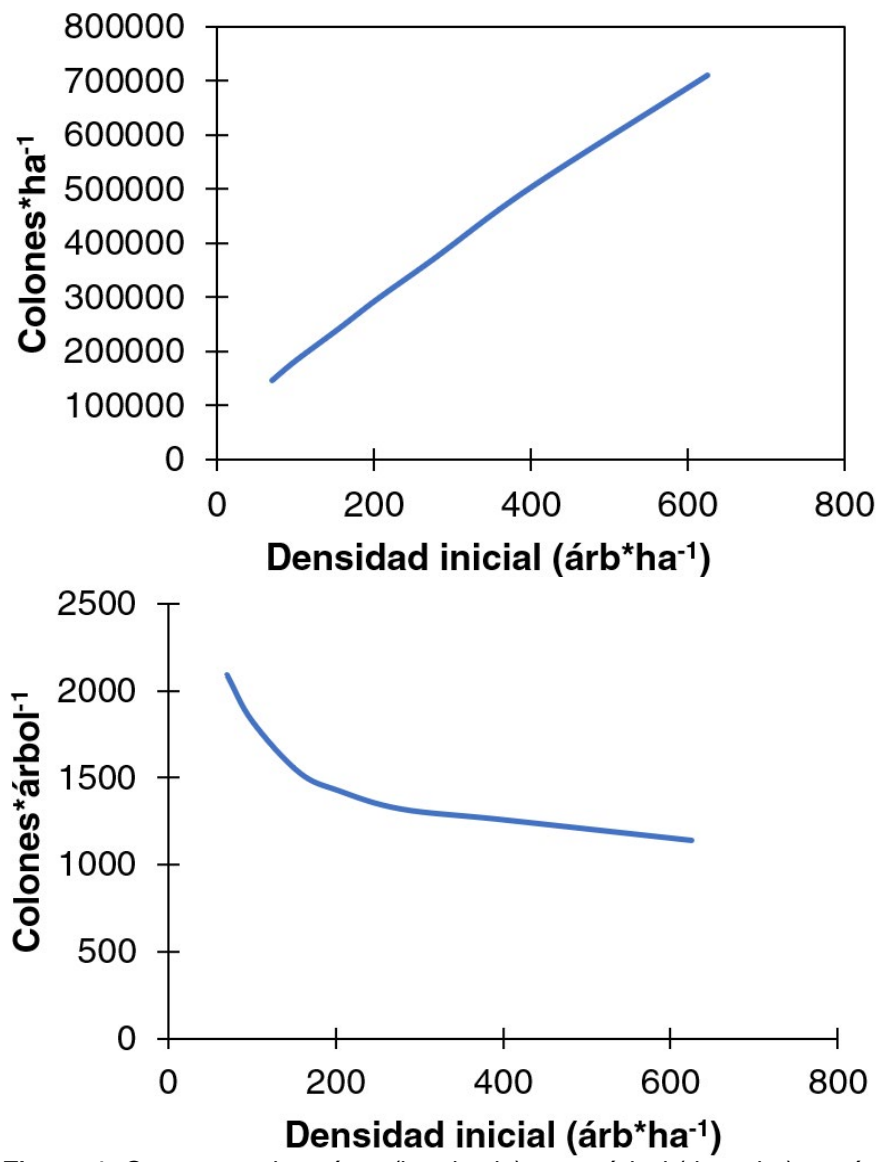

Figura 1. Costos por hectárea (izquierda) y por árbol (derecha) según densidad inicial de plantación

Figure 1. Hectare (left) and tree (right) cost according to initial plantation density.

del terreno, el análisis de suelo, control de arvenses, control de hormigas cortadoras y la fertilización anual se atribuyen al cultivo agrícola asociado. En los cuadros 6 ,
7 y 8 se presentan las estimaciones del costo del cultivo de caoba hasta los 4 años para estos sistemas.

El establecimiento representa un $62 \%$ del costo total y es significativamente más alto que cada uno de los costos anuales de manejo (Cuadro 6), ésta información es de suma importancia para planificar el flujo de caja y detallar el proyecto de inversión. Si se desea relacionar éstos resultados con la distribución anual de tractos de pago por servicios ambientales por parte del estado, es importante considerar que el establecimiento y el manejo del primer año representan un $71 \%$ de la inversión de los primeros 4 años, los que representan la etapa más crítica para él éxito del sistema de producción. En el cuarto año el costo es muy bajo ( $\left.\$ 4025^{*} \mathrm{ha}^{-1}\right)$ y corresponde al rubro de podas de las ramas que están en el fuste comercial.

En el análisis de cada rubro de costo, y en concordancia con lo mencionado anteriormente, las plantas y el control de $\mathrm{H}$. grandella representan los más elevados $(46,4 \%$ y $32,6 \%$ del costo total, respectivamente). De la misma manera que en los casos anteriores, una reducción en los costos de producción de los árboles clonados tendrá un efecto importante en la disminución de costos del establecimiento. En cuanto al control del barrenador, cuando el productor aporta la mano de obra de las fumigaciones, las erogaciones efectivas se reducen significativamente.

Por otra parte, es bien sabido que el costo de la mano de obra en las actividades forestales tiene un peso relativo significativo. Para éste caso es de \& $224000^{*} \mathrm{ha}^{-1} \mathrm{y}$ el de los materiales (incluyendo los árboles) es de \$ 254000 ha $^{-1}$, lo que significa un $42,4 \%$ y $50,4 \%$ del costo total, respectivamente (cuadro 8 ).

De la misma manera que con las fumigaciones, si el productor aporta lo que corresponde a mano de obra 
en general el costo total del establecimiento y manejo de la caoba como componente forestal del SAF se reduce hasta en un $42,4 \%$. De ésta manera se ratifica que ésta especie podría ser totalmente accesible tanto para pequeños como medianos productores que deseen diversificar mediante un componente de alto valor en sus fincas. El cual, además de los ingresos que le traerá en cada una de sus cosechas le brindará alta plusvalía a sus terrenos.

El Cuadro 9 presenta una proyección de los costos estimados, tanto por hectárea como por árbol, al considerar distintas densidades iniciales de plantación, con espaciamientos entre árboles que van desde $4 \mathrm{mx}$ $4 \mathrm{~m}$ hasta $12 \mathrm{~m} \times 12 \mathrm{~m}$. La selección del espaciamiento adecuado depende de las necesidades de luz o sombra de los cultivos asociados, del arreglo espacial y de la importancia relativa que se le dé a la producción de madera con respecto al cultivo asociado. Para cultivos perennes, como frutales, café, cacao, entre otros, los espaciamientos iguales o mayores a $8 \times 8$ son más utilizados.

Tal como se espera, el costo por hectárea decrece conforme disminuye el número de árboles plantados. Por el contrario, el costo por árbol aumenta rápidamente conforme disminuye la densidad inicial (Cuadro 9). Esto se debe en gran parte a los costos fijos, pero además a que los tiempos de recorrido entre árbol y árbol para realizar las diferentes labores tiene una relación directamente proporcional con el espaciamiento, es decir conforme aumenta el espaciamiento aumenta también el tiempo de recorrido entre árboles (Figura 1).

El costo por hectárea en este tipo de sistemas decrece desde $\$ 711679$ para el menor espaciamiento inicial reportado hasta $\$ 146665$ para el mayor (Figura 1). De forma inversa, el costo por árbol aumenta desde \$ 1139 para un espaciamiento de $4 \mathrm{~m} \times 4 \mathrm{~m}$ hasta $\$ 2095$ para 12 $\mathrm{m} \times 12 \mathrm{~m}$ (Figura 1). Para todas las densidades de siembra consideradas, tanto los costos por hectárea como por árbol siempre son menores que el costo promedio estimado para plantación pura, lo anterior porque existen actividades que le son cargadas al componente agrícola mediante éste sistema de producción.

En SAF, tanto por hectárea como por árbol, el costo de los materiales, los árboles y la capacitación inicial en conjunto representan un $59 \%$ y $53 \%$ del total, respectivamente; mientras que el costo de la mano de obra significa entre el $41 \%$ y $47 \%$ del total para la hectárea y el árbol. Cuando es el mismo productor el que aporta la mano de obra en proyectos pequeños, las erogaciones efectivas en que tiene que incurrir se reducen entre el $41 \%$ y $47 \%$. En dicho caso, los gastos efectivos oscilan entre $₫ 672^{*} a r^{-1}$ y $\$ 1121^{*}{ }^{*}$ rb $^{-1}$, según el espaciamiento utilizado, los que representa solo entre el $26 \%$ y $44 \%$ con respecto del costo en plantaciones puras con 625 árboles por hectárea.

Ligado a lo anterior, los resultados obtenidos sugieren que la combinación de árboles con cultivos agrícolas es la forma más económica de producir madera, para espaciamientos entre árboles iguales o menores a $12 \mathrm{~m}$ x $12 \mathrm{~m}$. Para una densidad inicial de 625 árboles $^{\star} \mathrm{ha}^{-1} \mathrm{el}$ costo estimado de una plantación pura es de $\$ 1598000$ *ha-1 ó \& 2556 *árb $^{-1}$ (sitio Horquetas, Cuadro 1), lo que representa un monto $125 \%$ mayor que el estimado para un sistema agroforestal (\$ $712000{ }^{*}$ ha $^{-1}$ ó \& 1139 *árb $^{-1}$ ).

\section{Conclusiones y recomendaciones}

El costo del cultivo de caoba en plantaciones puras es muy similar al de otras especies de alto valor como la teca. Para una densidad inicial de 625 árboles $^{*}$ ha $^{-1}$ oscila entre $\$ 1546000$ por hectárea y $\$ 1753000$ por hectárea (US\$ $2585-2930{ }^{*}$ ha $^{-1}$ ) y depende principalmente del clima del sitio y método de control de arvenses utilizado.

En sitios con estación seca marcada el costo es 6,8\% mayor que en sitios sin estación seca efectiva, por la necesidad de construir y mantener cortafuegos y utilizar retenedores de agua al momento de plantar.

El control del barrenador Hypsipyla grandella con insecticidas no representa en principio una limitante financiera muy importante para el cultivo de la caoba, representa un $13,6 \%$ el costo total para los cuatro primeros años del sistema de producción.

El cultivo de especies forestales en combinación con cultivos agrícolas es la forma más barata de producir madera, en contraposición con que el costo de producción hasta los cuatro años de edad en plantaciones puras es $125 \%$ mayor, desde luego con base en las condiciones bajo las que se realizó el presente estudio. No obstante, es necesario validar éstos resultados mediante plantaciones de clones superiores de caoba en distintas regiones del país, así como generar información sobre costos de producción de la caoba en sistemas silvopastoriles (SSP).

El costo por árbol en SAF hasta los cuatro años disminuye conforme aumenta la densidad inicial de siembra, registró valores entre \& 2095 y \& 1 139, para espaciamientos entre $4 \mathrm{~m} \times 4 \mathrm{~m}$ y $12 \mathrm{~m} \times 12 \mathrm{~m}$, respectivamente. 


\section{Referencias}

[1] X. García, B. Rodríguez y J. Islas, «Evaluación financiera de plantaciones forestales de caoba en Quintana Roo.,» Revista Mexicana de Ciencias Forestales, vol. 2, $n^{\circ}$ 7, pp. Págs. 7-26., 2011.

[2] E. Corea y O. Chinchilla, El cultivo de caoba (Swietenia macrophylla) en los primeros cuatro años de crecimiento, Costa Rica., Primera edición, Programa de publicaciones de la UNA,Heredia, Costa Rica., 2015, 25 p.

[3] O. Chinchilla, E. Corea y V. Meza, Silvicultura de clones supoeriores de caoba (Swietenia macrophylla) en los primeros cuatro años de crecimiento, Costa Rica., Tercera edición. ed., Heredia, Costa Rica: Instituto de Investigación y Servicios Forestales. Universidad Nacional., 2018, p. 38 p..

[4] L. Palma, "Costos de establecimiento, mantenimiento y manejo de plantaciones de teca (Tectona grandis L.f.) bajo sistemas intensivos de manejo, en Costa Rica", Trabajo Final de Graduación Bachillerato en Ingeniería en Ciencias Forestales, Escuela de Ciencias Ambientales. Universidad Nacional., Heredia, Costa Rica., 2017.

[5] L. Astúa, «Evaluación de las operaciones de mantenimiento mecanizado en plantaciones forestales Ecodirecta SA Los Chiles, Alajuela.,» Cartago, Costa Rica., 1999..

[6] R. Lujan, C. Herrera y D. Dipieri, «Plantaciones de teca en Panamá,» CATIE, Turrialba, Costa Rica., 2013.

[7] M. Gómez y C. Reiche, "Costos de establecimiento y manejo de plantaciones forestales y sistemas agroforestales en Costa Rica.,» CATIE/IICA/GIZ., Turrialba, Costa Rica., 1996.

[8] L. Sage, J. Kent y J. Morales, «Rentabilidad de las inversiones de teca.,» CATIE., Turrialba, Costa Rica., 2013.

[9] M. Guevara, «Productividad y costos operacionales de la chapea mecanizada en plantaciones jóves de Acacia mangium en la zona norte de Costa Rica., " Revista Forestal Mesoamericana Kurú., vol. 8, n² 20, pp. 27-30, 2011.

[10] INEC., «VI Censo Nacional Agropecuario: Resultados generales., » INEC., San José, Costa Rica., 2015.. 